\title{
Perturbative and non-perturbative renormalization results of the Chromomagnetic Operator on the Lattice
}

\author{
M. Constantinou ${ }^{a}$, M. Costa ${ }^{* a}$, R. Frezzotti ${ }^{b}$, V. Lubicz $^{c}$, G. Martinelli ${ }^{d}$, D. Meloni ${ }^{c}$, \\ H. Panagopoulos ${ }^{a}$, S. Simula ${ }^{c}$
}

${ }^{a}$ Department of Physics, University of Cyprus, CY-1678 Nicosia, Cyprus

${ }^{b}$ Dipartimento di Fisica, Università di Roma "Tor Vergata" and INFN Sezione "Tor Vergata", I-00133 Rome, Italy

${ }^{c}$ Dipartimento di Fisica, Università Roma Tre, and INFN, Sezione di Roma Tre, I-00146 Rome, Italy

${ }^{d}$ SISSA, I-34136 Trieste, Italy

E-mail: constantinou.martha@ucy.ac.cy, kosta.marios@ucy.ac.cy, roberto.frezzottieroma2.infn.it, lubiczefis.uniroma3.it, guido.martinelli@sissa.it, meloni@fis.uniroma3.it, haris@ucy.ac.cy, simula@roma3.infn.it

The Chromomagnetic operator (CMO) mixes with a large number of operators under renormalization. We identify which operators can mix with the $\mathrm{CMO}$, at the quantum level. Even in dimensional regularization (DR), which has the simplest mixing pattern, the CMO mixes with a total of 9 other operators, forming a basis of dimension-five, Lorentz scalar operators with the same flavor content as the CMO. Among them, there are also gauge noninvariant operators; these are BRST invariant and vanish by the equations of motion, as required by renormalization theory. On the other hand using a lattice regularization further operators with $d \leq 5$ will mix; choosing the lattice action in a manner as to preserve certain discrete symmetries, a minimul set of 3 additional operators (all with $d<5$ ) will appear. In order to compute all relevant mixing coefficients, we calculate the quark-antiquark (2-pt) and the quark-antiquark-gluon (3-pt) Green's functions of the CMO at nonzero quark masses. These calculations were performed in the continuum (dimensional regularization) and on the lattice using the maximally twisted mass fermion action and the Symanzik improved gluon action. In parallel, non-perturbative measurements of the $K-\pi$ matrix element are being performed in simulations with 4 dynamical $\left(N_{f}=2+1+1\right)$ twisted mass fermions and the Iwasaki improved gluon action.

32nd International Symposium on Lattice Field Theory LATTICE 2014

June 23 - June 28, 2014

New York, USA

\footnotetext{
* Speaker.
} 


\section{Definition of the Chromomagnetic operator}

We study the mixing of the chromomagnetic operator (CMO):

$$
\mathscr{O}_{C M}=g_{0} \bar{\psi}_{s} \sigma_{\mu \nu} G_{\mu \nu} \psi_{d},
$$

using both dimensional regularization (DR) and lattice regularization (L). The CMO appears naturally, via the OPE, in an effective Hamiltonian description of semi-leptonic processes. For $\Delta S=1$ transitions, the low-energy effective Hamiltonian $H_{\text {eff }}$ contains four magnetic operators of dimension 5:

$$
\begin{gathered}
H_{\mathrm{eff}}^{\Delta S=1, d=5}=\sum_{i= \pm}\left(C_{\gamma}^{i}(\mu) Q_{\gamma}^{i}(\mu)+C_{g}^{i}(\mu) Q_{g}^{i}(\mu)\right)+\text { h.c. } \\
Q_{\gamma}^{ \pm}=\frac{Q_{d} e}{16 \pi^{2}}\left(\bar{\psi}_{s L} \sigma^{\mu v} F_{\mu \nu} \psi_{d R} \pm \bar{\psi}_{s R} \sigma^{\mu v} F_{\mu v} \psi_{d L}\right), Q_{g}^{ \pm}=\frac{g}{16 \pi^{2}}\left(\bar{\psi}_{s L} \sigma^{\mu v} G_{\mu v} \psi_{d R} \pm \bar{\psi}_{s R} \sigma^{\mu v} G_{\mu v} \psi_{d L}\right)
\end{gathered}
$$

Some of the matrix elements of the CMO are parameterized as [2] :

$$
\begin{gathered}
\left\langle\pi^{0}\left|Q_{g}^{+}\right| K^{0}\right\rangle=\frac{-11}{32 \sqrt{2} \pi^{2}} \frac{M_{K}^{2}\left(p_{\pi} \cdot p_{K}\right)}{m_{s}+m_{d}} B_{g 1}, \quad\left\langle\pi^{+} \pi^{-}\left|Q_{g}^{-}\right| K^{0}\right\rangle=\frac{11 \mathrm{i}}{32 \pi^{2}} \frac{M_{K}^{2} M_{\pi}^{2}}{f_{\pi}\left(m_{s}+m_{d}\right)} B_{g 2} \\
\left\langle\pi^{+} \pi^{+} \pi^{-}\left|Q_{g}^{+}\right| K^{+}\right\rangle=\frac{-11}{16 \pi^{2}} \frac{M_{K}^{2} M_{\pi}^{2}}{f_{\pi}^{2}\left(m_{s}+m_{d}\right)} B_{g 3} .
\end{gathered}
$$

These matrix elements are relevant for the study of $K^{0}-\bar{K}^{0}$ mixing, $\varepsilon^{\prime} / \varepsilon$, the $\Delta I=1 / 2$ rule, and $K \rightarrow 3 \pi$ decays. We focus on the matrix elements of $\mathscr{O}_{C M}$ between a kaon and a pion state. The $K-\pi$ matrix element of $\mathscr{O}_{C M}$ has never been calculated before on the lattice. The main difficulty of lattice calculation of the matrix elements of the $\mathrm{CMO}$ is that the strong interactions induce a mixing of the CMO with operators of lower dimension, with coefficients which are power divergent with the cutoff. The leading divergence of the bare CMO, which is of order $1 / a^{2}$, is determined by the mixing with the dimension 3 scalar operator $\bar{\psi}_{s} \psi_{d}$. Being power divergent, this subtraction is numerically difficult to control. In addition, its coefficient must be eventually evaluated in a fully non-perturbative way, since non-perturbative effects, e.g., factors of the form $a \Lambda_{Q C D}$, combined with factors which diverge as inverse powers of the lattice spacing can give finite contributions [3].

\section{Symmetries of the Action and Transformation Properties of operators}

With our choice of lattice action, there exist certain symmetries of the action (valid both in the continuum and lattice formulation of the theory) which reduce considerably the number of operators that can possibly mix with $\mathscr{O}_{C M}$ at the quantum level. These symmetries are defined by means of the discrete transformations $\mathscr{P}$ (continuum parity), $\mathscr{D}_{d}, \mathscr{R}_{5}$ [1], $\mathscr{C}$ (charge conjugation), and $\mathscr{S}$ (exchange between the $s$ and the $d$ quark). In terms of the above transformations, the symmetries of the action are:

$$
\begin{aligned}
& \text { - } \mathscr{P} \times \mathscr{D}_{d} \times(m \rightarrow-m) \text {, where } m \text { are all masses except } M_{\mathrm{cr}} \\
& \text { - } \mathscr{D}_{d} \times \mathscr{R}_{5} \\
& \text { - } \mathscr{C} \times \mathscr{S} \text {, if } r_{s}=r_{d} \\
& \text { - } \mathscr{C} \times \mathscr{P} \times \mathscr{S} \text {, if } r_{s}=-r_{d} .
\end{aligned}
$$

In order to identify which operators can possibly mix with $\mathscr{O}_{C M}$, we examine the transformation properties of all candidate operators under the above symmetries; admissible operators must transform in the same way as $\mathscr{O}_{C M}$. Furthermore, by general renormalization theorems, these operators 


\begin{tabular}{|c|c|c|c|c|c|}
\hline \multicolumn{2}{|c|}{ Operators } & $\begin{array}{l}\mathscr{P} \times \mathscr{D}_{d} \times \\
(m \rightarrow-m) \\
\end{array}$ & $\mathscr{D}_{d} \times \mathscr{R}_{5}$ & $\begin{array}{c}\mathscr{C} \times \mathscr{S} \\
\text { if } r_{s}=r_{d}\end{array}$ & $\begin{array}{c}\mathscr{C} \times \mathscr{P} \times \mathscr{S} \\
\text { if } r_{s}=-r_{d} \\
\end{array}$ \\
\hline \multicolumn{6}{|c|}{ Dimension 3 operators } \\
\hline$\checkmark$ & $\begin{array}{l}\bar{\psi}_{s} \psi_{d} \\
i \bar{\psi}_{s} \gamma_{5} \psi_{d}\end{array}$ & $\begin{array}{l}- \\
+\end{array}$ & $\begin{array}{l}+ \\
+\end{array}$ & $\begin{array}{l}+ \\
+\end{array}$ & $\begin{array}{l}+ \\
-\end{array}$ \\
\hline \multicolumn{6}{|c|}{ Dimension 4 operators } \\
\hline $\begin{array}{l}(+) \\
(-)\end{array}$ & $\begin{array}{l}\left(m_{d}+m_{s}\right) \bar{\psi}_{s} \psi_{d} \\
\left(m_{d}-m_{s}\right) \bar{\psi}_{s} \psi_{d} \\
i\left(m_{d}+m_{s}\right) \bar{\psi}_{s} \gamma_{5} \psi_{d} \\
i\left(m_{d}-m_{s}\right) \bar{\psi}_{s} \gamma_{5} \psi_{d} \\
\bar{\psi}_{s}\left(\vec{D}+m_{d}\right) \psi_{d}+\bar{\psi}_{s}\left(-\overleftarrow{D}+m_{s}\right) \psi_{d} \\
\bar{\psi}_{s}\left(\vec{D}+m_{d}\right) \psi_{d}-\bar{\psi}_{s}\left(-\overleftarrow{D}+m_{s}\right) \psi_{d} \\
i \bar{\psi}_{s} \gamma_{5}\left(\vec{D}+m_{d}\right) \psi_{d}+i \bar{\psi}_{s}\left(-\overleftarrow{D}+m_{s}\right) \gamma_{5} \psi_{d} \\
i \bar{\psi}_{s} \gamma_{5}\left(\vec{D}+m_{d}\right) \psi_{d}-i \bar{\psi}_{s}\left(-\overleftarrow{D}+m_{s}\right) \gamma_{5} \psi_{d}\end{array}$ & $\begin{array}{l}+ \\
+ \\
- \\
- \\
+ \\
+ \\
- \\
-\end{array}$ & $\begin{array}{l}+ \\
+ \\
+ \\
+ \\
+ \\
+ \\
+ \\
+\end{array}$ & $\begin{array}{l}+ \\
- \\
+ \\
- \\
+ \\
- \\
+ \\
-\end{array}$ & $\begin{array}{l}+ \\
- \\
- \\
+ \\
+ \\
- \\
-\end{array}$ \\
\hline
\end{tabular}

Table 1: Transformation properties of dimension 3 and 4 operators. Gauge non-invariant operators do not appear, since they do not vanish by the eom.

must be gauge invariant, or else they must vanish by the equations of motion (eom). In Tables 1 and 2 we present all candidate operators along with their transformation properties. Operators marked by " $\checkmark$ " have the same properties as $\mathscr{O}_{C M}$ and thus may mix with it. Operators marked by " $(+)^{\prime \prime}$ or " $(-)^{\prime \prime}$ have the same transformation properties as $\mathscr{O}_{C M}$ only if $r_{s}=r_{d}$ or $r_{s}=-r_{d}$, respectively; for this reason the Wilson parameters $r_{s}, r_{d}$ have been explicitly introduced in $\mathscr{O}_{11}$ and $\mathscr{O}_{12}$ below (see Eqs. (2.6) - (2.6)). There follows immediately that $\mathscr{O}_{C M} \equiv \mathscr{O}_{1}$ can only mix with the following operators:

$$
\begin{aligned}
& \mathscr{O}_{1}=g_{0} \bar{\psi}_{s} \sigma_{\mu \nu} G_{\mu \nu} \psi_{d}, \quad \mathscr{O}_{2}=\left(m_{d}^{2}+m_{s}^{2}\right) \bar{\psi}_{s} \psi_{d}, \quad \mathscr{O}_{3}=m_{d} m_{s} \bar{\psi}_{s} \psi_{d}, \quad \mathscr{O}_{4}=\square\left(\bar{\psi}_{s} \psi_{d}\right) \\
& \mathscr{O}_{5}=\bar{\psi}_{s}\left(-\overleftarrow{D}+m_{s}\right)\left(\vec{D}+m_{d}\right) \psi_{d}, \quad \mathscr{O}_{6}=\bar{\psi}_{s}\left(\vec{D}+m_{d}\right)^{2} \psi_{d}+\bar{\psi}_{s}\left(-\overleftarrow{D}+m_{s}\right)^{2} \psi_{d} \\
& \mathscr{O}_{7}=m_{s} \bar{\psi}_{s}\left(\vec{D}+m_{d}\right) \psi_{d}+m_{d} \bar{\psi}_{s}\left(-\overleftarrow{D}+m_{s}\right) \psi_{d}, \mathscr{O}_{8}=m_{d} \bar{\psi}_{s}\left(\vec{D}+m_{d}\right) \psi_{d}+m_{s} \bar{\psi}_{s}\left(-\overleftarrow{D}+m_{s}\right) \psi_{d} \\
& \mathscr{O}_{9}=\bar{\psi}_{s} \overleftarrow{\not}\left(\overrightarrow{\not D}+m_{d}\right) \psi_{d}-\bar{\psi}_{s}\left(-\overleftarrow{D}+m_{s}\right) \vec{\not} \psi_{d}, \mathscr{O}_{10}=\bar{\psi}_{s} \vec{\not}\left(\vec{D}+m_{d}\right) \psi_{d}-\bar{\psi}_{s}\left(-\overleftarrow{\not}+m_{s}\right) \overleftarrow{\not} \psi_{d} \\
& \mathscr{O}_{11}=i r_{d} \bar{\psi}_{s} \gamma_{5}\left(\vec{D}+m_{d}\right) \psi_{d}+i r_{s} \bar{\psi}_{s}\left(-\overleftarrow{D}+m_{s}\right) \gamma_{5} \psi_{d}, \quad \mathscr{O}_{12}=i\left(r_{d} m_{d}+r_{s} m_{s}\right) \bar{\psi}_{s} \gamma_{5} \psi_{d} \\
& \mathscr{O}_{13}=\bar{\psi}_{s} \psi_{d} \text {. }
\end{aligned}
$$

For the parameters $r_{s}, r_{d}$, in our perturbative calculation we have made the (independent) choices of values $r_{s}= \pm 1, r_{d}= \pm 1$, consistently with their values in simulations. Operators $\mathscr{O}_{9}$ and $\mathscr{O}_{10}$ are not gauge invariant, but they vanish by the eom; indeed, they will mix with $\mathscr{O}_{C M}$ both in dimensional regularization and on the lattice. The operators $\mathscr{O}_{11}, \mathscr{O}_{12}, \mathscr{O}_{13}$ are of lower dimension and thus they do not mix with $\mathscr{O}_{1}$ in dimensional regularization; they do however show up in the lattice formulation. The renormalized operators $\mathscr{O}_{i}^{R}$ are related to the bare ones, $\mathscr{O}_{i}(i=1, \ldots, 13)$, through:

$$
\mathscr{O}_{i}=\sum_{j=1}^{13} Z_{i j} \mathscr{O}_{j}^{R} \quad\left(\text { in matrix notation }: \mathscr{O}=Z \mathscr{O}^{R}\right)
$$

where $Z_{i j}$ is a $13 \times 13$ mixing matrix. For $\mathscr{O}_{1}^{R}$, we only need the first row of $Z^{-1}$. The bare 


\begin{tabular}{|c|c|c|c|c|c|}
\hline \multicolumn{2}{|r|}{ Dimension 5 Operators } & \multirow{2}{*}{$\begin{array}{c}\mathscr{P} \times \mathscr{D}_{d} \times \\
\frac{(m \rightarrow-m)}{-}\end{array}$} & \multirow{2}{*}{$\begin{array}{c}\mathscr{D}_{d} \times \mathscr{R}_{5} \\
+\end{array}$} & \multirow{2}{*}{$\begin{array}{c}\mathscr{C} \times \mathscr{S} \\
\text { if } r_{s}=r_{d} \\
+\end{array}$} & \multirow{2}{*}{$\begin{array}{c}\mathscr{C} \times \mathscr{P} \times \mathscr{S} \\
\text { if } r_{s}=-r_{d} \\
+\end{array}$} \\
\hline$\checkmark$ & $g_{0} \bar{\psi}_{s} \sigma_{\mu \nu} G_{\mu \nu} \psi_{d}$ & & & & \\
\hline & $i g_{0} \bar{\psi}_{s} \gamma_{5} \sigma_{\mu v} G_{\mu v} \psi_{d}$ & + & + & + & - \\
\hline$\checkmark$ & $\left(m_{d}^{2}+m_{s}^{2}\right) \bar{\psi}_{s} \psi_{d}$ & - & + & + & + \\
\hline & $i\left(m_{d}^{2}+m_{s}^{2}\right) \bar{\psi}_{s} \gamma_{5} \psi_{d}$ & + & + & + & - \\
\hline & $\left(m_{d}^{2}-m_{s}^{2}\right) \bar{\psi}_{s} \psi_{d}$ & - & + & - & - \\
\hline & $i\left(m_{d}^{2}-m_{s}^{2}\right) \bar{\psi}_{s} \gamma_{5} \psi_{d}$ & + & + & - & + \\
\hline$\checkmark$ & $m_{d} m_{s} \bar{\psi}_{s} \psi_{d}$ & - & + & + & + \\
\hline & $i m_{d} m_{s} \bar{\psi}_{s} \gamma_{5} \psi_{d}$ & + & + & + & - \\
\hline$\checkmark$ & $m_{s} \bar{\psi}_{s}\left(\vec{D}+m_{d}\right) \psi_{d}+m_{d} \bar{\psi}_{s}\left(-\overleftarrow{\not D}+m_{s}\right) \psi_{d}$ & - & + & + & + \\
\hline$\checkmark$ & $m_{d} \bar{\psi}_{s}\left(\vec{D}+m_{d}\right) \psi_{d}+m_{s} \bar{\psi}_{s}\left(-\overleftarrow{D}+m_{s}\right) \psi_{d}$ & - & + & + & + \\
\hline & $m_{s} \bar{\psi}_{s}\left(\vec{D}+m_{d}\right) \psi_{d}-m_{d} \bar{\psi}_{s}\left(-\overleftarrow{\not D}+m_{s}\right) \psi_{d}$ & - & + & - & - \\
\hline & $m_{d} \bar{\psi}_{s}\left(\vec{D}+m_{d}\right) \psi_{d}-m_{s} \bar{\psi}_{s}\left(-\overleftarrow{D}+m_{s}\right) \psi_{d}$ & - & + & - & - \\
\hline & $i m_{s} \bar{\psi}_{s} \gamma_{5}\left(\vec{D}+m_{d}\right) \psi_{d}+i m_{d} \bar{\psi}_{s}\left(-\overleftarrow{D}+m_{s}\right) \gamma_{5} \psi_{d}$ & + & + & + & - \\
\hline & $i m_{d} \bar{\psi}_{s} \gamma_{5}\left(\vec{D}+m_{d}\right) \psi_{d}+i m_{s} \bar{\psi}_{s}\left(-\overleftarrow{\not D}+m_{s}\right) \gamma_{5} \psi_{d}$ & + & + & + & - \\
\hline & $i m_{s} \bar{\psi}_{s} \gamma_{5}\left(\vec{D}+m_{d}\right) \psi_{d}-i m_{d} \bar{\psi}_{s}\left(-\overleftarrow{D}+m_{s}\right) \gamma_{5} \psi_{d}$ & + & + & - & + \\
\hline & $i m_{d} \bar{\psi}_{s} \gamma_{5}\left(\vec{D}+m_{d}\right) \psi_{d}-i m_{s} \bar{\psi}_{s}\left(-\overleftarrow{D}+m_{s}\right) \gamma_{5} \psi_{d}$ & + & + & - & + \\
\hline$\checkmark$ & $\bar{\psi}_{s}\left(\vec{D}+m_{d}\right)^{2} \psi_{d}+\bar{\psi}_{s}\left(-\overleftarrow{D}+m_{s}\right)^{2} \psi_{d}$ & - & + & + & + \\
\hline & $\bar{\psi}_{s}\left(\vec{D}+m_{d}\right)^{2} \psi_{d}-\bar{\psi}_{s}\left(-\overleftarrow{\not D}+m_{s}\right)^{2} \psi_{d}$ & - & + & - & - \\
\hline & $i \bar{\psi}_{s} \gamma_{5}\left(\vec{D}+m_{d}\right)^{2} \psi_{d}+i \bar{\psi}_{s}\left(-\overleftarrow{\not D}+m_{s}\right)^{2} \gamma_{5} \psi_{d}$ & + & + & + & - \\
\hline & $i \bar{\psi}_{s} \gamma_{5}\left(\vec{D}+m_{d}\right)^{2} \psi_{d}-i \bar{\psi}_{s}\left(-\overleftarrow{\not D}+m_{s}\right)^{2} \gamma_{5} \psi_{d}$ & + & + & - & + \\
\hline$\checkmark$ & $\bar{\psi}_{s} \overleftarrow{D}_{\mu} \vec{D}_{\mu} \psi_{d}$ & - & + & + & + \\
\hline & $i \bar{\psi}_{s} \gamma_{5} \overleftarrow{D}_{\mu} \vec{D}_{\mu} \psi_{d}$ & + & + & + & - \\
\hline$\checkmark$ & $\bar{\psi}_{s}\left(-\overleftarrow{D}+m_{s}\right)\left(\vec{D}+m_{d}\right) \psi_{d}$ & - & + & + & + \\
\hline & $i \bar{\psi}_{s}\left(-\overleftarrow{D}+m_{s}\right) \gamma_{5}\left(\vec{D}+m_{d}\right) \psi_{d}$ & + & + & + & - \\
\hline$\checkmark$ & $\bar{\psi}_{s} \overleftarrow{\not}\left(\vec{D}+m_{d}\right) \psi_{d}-\bar{\psi}_{s}\left(-\overleftarrow{\not D}+m_{s}\right) \vec{\not} \psi_{d}$ & - & + & + & + \\
\hline$\checkmark$ & $\bar{\psi}_{s} \vec{\partial}\left(\vec{D}+m_{d}\right) \psi_{d}-\bar{\psi}_{s}\left(-\overleftarrow{D}+m_{s}\right) \overleftarrow{\partial} \psi_{d}$ & - & + & + & + \\
\hline & $\bar{\psi}_{s} \overleftarrow{\not}\left(\vec{D}+m_{d}\right) \psi_{d}+\bar{\psi}_{s}\left(-\overleftarrow{\not D}+m_{s}\right) \vec{\partial} \psi_{d}$ & - & + & - & - \\
\hline & $\bar{\psi}_{s} \overrightarrow{\not \partial}\left(\vec{D}+m_{d}\right) \psi_{d}+\bar{\psi}_{s}\left(-\overleftarrow{D}+m_{s}\right) \overleftarrow{\not} \psi_{d}$ & - & + & - & - \\
\hline & $i \bar{\psi}_{s} \overleftarrow{\partial} \gamma_{5}\left(\vec{D}+m_{d}\right) \psi_{d}-i \bar{\psi}_{s}\left(-\overleftarrow{D}+m_{s}\right) \gamma_{5} \vec{\partial} \psi_{d}$ & + & + & + & - \\
\hline & $i \bar{\psi}_{s} \vec{\partial} \gamma_{5}\left(\vec{D}+m_{d}\right) \psi_{d}-i \bar{\psi}_{s}\left(-\overleftarrow{D}+m_{s}\right) \gamma_{5} \overleftarrow{\not \partial} \psi_{d}$ & + & + & + & - \\
\hline & $i \bar{\psi}_{s} \overleftarrow{\partial} \gamma_{5}\left(\vec{D}+m_{d}\right) \psi_{d}+i \bar{\psi}_{s}\left(-\overleftarrow{\not D}+m_{s}\right) \gamma_{5} \vec{\partial} \psi_{d}$ & + & + & - & + \\
\hline & $i \bar{\psi}_{s} \vec{\partial} \gamma_{5}\left(\vec{D}+m_{d}\right) \psi_{d}+i \bar{\psi}_{s}\left(-\overleftarrow{D}+m_{s}\right) \gamma_{5} \overleftarrow{\partial} \psi_{d}$ & + & + & - & + \\
\hline
\end{tabular}

Table 2: Transformation properties of gauge invariant operators and of operators which vanish by the equations of motion, in the physical basis (Operator dimension $=5$ ). 
amputated Green's functions of $\mathscr{O}_{1}$ are related to the corresponding renormalized ones through:

$$
\begin{gathered}
\left\langle\psi^{R} \mathscr{O}_{1}^{R} \bar{\psi}^{R}\right\rangle_{\mathrm{amp}}=Z_{\psi} \sum_{i=1}^{13}\left(Z^{-1}\right)_{1 i}\left\langle\psi \mathscr{O}_{i} \bar{\psi}\right\rangle_{\mathrm{amp}}, \quad \psi=\sqrt{Z_{\psi}} \psi^{R} \\
\left\langle\psi^{R} \mathscr{O}_{1}^{R} \bar{\psi}^{R} A_{v}^{R}\right\rangle_{\mathrm{amp}}=Z_{\psi} Z_{A}^{1 / 2} \sum_{i=1}^{13}\left(Z^{-1}\right)_{1 i}\left\langle\psi \mathscr{O}_{i} \bar{\psi} A_{v}\right\rangle_{\mathrm{amp}}, \quad A_{v}=\sqrt{Z_{A}} A_{v}^{R}
\end{gathered}
$$

\section{Lattice regularization - Renormalization functions in the $\overline{\mathrm{MS}}$ scheme}

The general form of the divergent (logarithmic) part of the bare Green's functions is:

$$
\begin{aligned}
& \left.\left\langle\psi \mathscr{O}_{1} \bar{\psi}\right\rangle_{\operatorname{amp}}^{L}\right|_{\log \left(a^{2}\right)}=\rho_{1}\left(q_{s}^{2}+q_{d}^{2}\right)+\rho_{2}\left(m_{s}^{2}+m_{d}^{2}\right)+\rho_{3} i\left(m_{d} q_{d}+m_{s} q_{s}\right)+\rho_{4} i\left(m_{s} q_{d}+m_{d} q_{s}\right) \\
& +\rho_{5} q_{s} \cdot q_{d}+\rho_{6} q_{s} q_{d}+\rho_{7} m_{s} m_{d}+\rho_{8}\left(r_{d} \gamma_{5} q_{d}+r_{s} q_{s} \gamma_{5}\right) \\
& +\rho_{9} i\left(r_{d} m_{d}+r_{s} m_{s}\right) \gamma_{5}+\rho_{10} \cdot 1 \\
& \left.\left\langle\psi \mathscr{O}_{1} \bar{\psi} A_{v}\right\rangle_{\mathrm{amp}, 1 P I}^{L}\right|_{\log \left(a^{2}\right)}=R_{1} g\left(q_{s}+q_{d}\right)_{v}+R_{2} g\left(\gamma_{v} q_{d}+q_{s} \gamma_{v}\right)+R_{3} i g\left(m_{s}+m_{d}\right) \gamma_{v} \\
& +R_{4}\left(-2 i g \sigma_{\rho v} q_{A \rho}\right)+R_{5} g\left(r_{d}-r_{s}\right) \gamma_{5} \gamma_{v}
\end{aligned}
$$

where $\rho_{i}, R_{i}$ are numerical coefficients and $q_{s} / q_{d} / q_{A}$ is the momentum of the external antiquark/quark/gluon. The MS-renormalized Green's functions are already known in dimensional regularization, see Ref. [4]. Equations (2.9) and (2.10) now take the form:

$$
\begin{gathered}
\left\langle\psi \mathscr{O}_{1} \bar{\psi}\right\rangle_{\mathrm{amp}}^{\overline{\mathrm{MS}}}=Z_{\psi}^{L, \overline{\mathrm{MS}}} \sum_{i=1}^{13}\left(\left(Z^{L, \overline{\mathrm{MS}}}\right)^{-1}\right)_{1 i}\left\langle\psi \mathscr{O}_{i} \bar{\psi}\right\rangle_{\mathrm{amp}}^{L}, \\
\left\langle\psi \mathscr{O}_{1} \bar{\psi} A_{v}\right\rangle_{\mathrm{amp}}^{\overline{\mathrm{MS}}}=Z_{\psi}^{L, \overline{\mathrm{MS}}}\left(Z_{A}^{L, \overline{\mathrm{MS}}}\right)^{1 / 2} \sum_{i=1}^{13}\left(\left(Z^{L, \overline{\mathrm{MS}}}\right)^{-1}\right)_{1 i}\left\langle\psi \mathscr{O}_{i} \bar{\psi} A_{v}\right\rangle_{\mathrm{amp}}^{L} .
\end{gathered}
$$

Renormalizability of the theory implies that the difference between the one-loop renormalized and bare Green's functions must only consist of expressions which are polynomial in $q_{i}, m$ having the form shown in Eqs. (3.1) - (3.2); in this way, the right-hand sides of Eqs. (3.3) - (3.4) can be rendered equal to the corresponding left-hand sides, by an appropriate definition of the $\left(q_{i^{-}}\right.$ and $m$-independent) renormalization functions $Z_{1 i}^{L, \overline{\mathrm{MS}}} \equiv Z_{i}^{L, \overline{\mathrm{MS}}}$. These differences can be written as follows:

$$
\begin{gathered}
\left\langle\psi \mathscr{O}_{1} \bar{\psi}\right\rangle_{\mathrm{amp}}^{\overline{\mathrm{MS}}}-\left\langle\psi \mathscr{O}_{1} \bar{\psi}\right\rangle_{\mathrm{amp}}^{L}=g^{2}\left(z_{\psi}^{L, \overline{\mathrm{MS}}}+z_{g}^{L, \overline{\mathrm{MS}}}-z_{1}^{L, \overline{\mathrm{MS}}}\right)\left\langle\psi \mathscr{O}_{1} \bar{\psi}\right\rangle_{\text {tree }}-\sum_{i=2}^{13} Z_{i}^{L, \overline{\mathrm{MS}}}\left\langle\psi \mathscr{O}_{i} \bar{\psi}\right\rangle_{\text {tree }} \\
\left\langle\psi \mathscr{O}_{1} \bar{\psi} A_{v}\right\rangle_{\mathrm{amp}_{\mathrm{am}}}^{\overline{\mathrm{MS}}}-\left\langle\psi \mathscr{O}_{1} \bar{\psi} A_{v}\right\rangle_{\mathrm{amp}}^{L}=g^{2}\left(z_{\psi}^{L, \overline{\mathrm{MS}}}+\frac{1}{2} z_{A}^{L, \overline{\mathrm{MS}}}+z_{g}^{L, \overline{\mathrm{MS}}}-z_{1}^{L, \overline{\mathrm{MS}}}\right)\left\langle\psi \mathscr{O}_{1} \bar{\psi} A_{v}\right\rangle_{\text {tree }}-\sum_{i=2}^{13} z_{i}^{L, \overline{\mathrm{MS}}}\left\langle\psi \mathscr{O}_{i} \bar{\psi} A_{v}\right\rangle_{\text {tree }}, \\
\text { where } Z_{1}^{L, \overline{\mathrm{MS}}}=1+g^{2} z_{1}^{L, \overline{\mathrm{MS}}}+\mathscr{O}\left(g^{4}\right), Z_{i>1}^{L, \overline{\mathrm{MS}}}=\mathscr{O}\left(g^{2}\right) \text { and } Z_{\psi}^{L, \overline{\mathrm{MS}}}=1+g^{2} z_{\psi}^{L, \overline{\mathrm{MS}}}+\mathscr{O}\left(g^{4}\right), Z_{A}^{L, \overline{\mathrm{MS}}}= \\
1+g^{2} z_{A}^{L, \overline{\mathrm{MS}}}+\mathscr{O}\left(g^{4}\right), \quad Z_{g}^{L, \overline{\mathrm{MS}}}=1+g^{2} z_{g}^{L, \overline{\mathrm{MS}}}+\mathscr{O}\left(g^{4}\right) .
\end{gathered}
$$

Equations (3.5) and (3.6) amount to $10+5$ conditions for the 13 mixing coefficients; they are consistent and admit a unique solution. Upon solving them we obtain for the Iwasaki gluon action:

$$
Z_{1}^{L, \overline{\mathrm{MS}}}=1+\frac{g^{2}}{16 \pi^{2}}\left(N_{c}\left(-7.9438+\frac{1}{2} \log \left(a^{2} \bar{\mu}^{2}\right)\right)+\frac{1}{N_{c}}\left(4.4851-\frac{5}{2} \log \left(a^{2} \bar{\mu}^{2}\right)\right)\right)
$$




$$
\begin{aligned}
& Z_{2}^{L, \overline{\mathrm{MS}}}=\frac{g^{2} C_{F}}{16 \pi^{2}}\left(4.5370+6 \log \left(a^{2} \bar{\mu}^{2}\right)\right), \quad Z_{3}^{L, \overline{\mathrm{MS}}}=Z_{4}^{L, \overline{\mathrm{MS}}}=0 \\
& Z_{5}^{L, \overline{\mathrm{MS}}}=\frac{g^{2}}{16 \pi^{2}}\left(N_{c}\left(4.2758-\frac{3}{2} \log \left(a^{2} \bar{\mu}^{2}\right)\right)+\frac{1}{N_{c}}\left(-3.7777+3 \log \left(a^{2} \bar{\mu}^{2}\right)\right)\right) \\
& Z_{6}^{L, \overline{\mathrm{MS}}}=0, \quad Z_{7}^{L, \overline{\mathrm{MS}}}=-\frac{Z_{5}^{L, \overline{\mathrm{MS}}}}{2}, \quad Z_{8}^{L, \overline{\mathrm{MS}}}=\frac{g^{2} C_{F}}{16 \pi^{2}}(-3.7760) \\
& Z_{9}^{L, \overline{\mathrm{MS}}}=\frac{Z_{5}^{L, \overline{\mathrm{MS}}}}{2}, \quad Z_{10}^{L, \overline{\mathrm{MS}}}=\frac{g^{2} C_{F}}{16 \pi^{2}}\left(3.7777-3 \log \left(a^{2} \bar{\mu}^{2}\right)\right) \\
& Z_{11}^{L, \overline{\mathrm{MS}}}=\frac{1}{a} \frac{g^{2} C_{F}}{16 \pi^{2}}(-3.2020), \quad Z_{12}^{L, \overline{\mathrm{MS}}}=-Z_{11}^{L, \overline{\mathrm{MS}}}, \quad Z_{13}^{L, \overline{\mathrm{MS}}}=\frac{1}{a^{2}} \frac{g^{2} C_{F}}{16 \pi^{2}} \text { (36.0613) . }
\end{aligned}
$$

For the operators of lower dimensionality $\left(\mathscr{O}_{11}-\mathscr{O}_{13}\right)$, given that their coefficients are power divergent, perturbation theory is expected to provide only a ballpark estimate at best. Fortunately, it is precisely for the coefficients of these latter operators that we can have best access to nonperturbative estimates.

\section{Non-perturbative results}

In this Section we present our non-perturbative determination of the coefficient $Z_{13}$ describing the power-divergent mixing of the chromomagnetic operator with the scalar density $\left(\mathscr{O}_{13}\right)$. We use lattice QCD simulations with the gauge configurations produced by ETMC with four flavors of dynamical quarks $\left(N_{f}=2+1+1\right)$, which include in the sea, besides two light mass degenerate quarks, also the strange and charm quarks with masses close to their physical values. We impose non-perturbative conditions:

$$
\begin{aligned}
& \lim _{m_{s}, m_{d} \rightarrow 0}\left\langle\pi(0)\left|\mathscr{O}_{1}^{\mathrm{R}}\right| K(0)\right\rangle=\lim _{m_{s}, m_{d} \rightarrow 0}\left\langle\pi(0)\left|\mathscr{O}_{1}-\frac{c_{13}}{a^{2}} \mathscr{O}_{13}\right| K(0)\right\rangle=0 \\
& \left\langle 0\left|\mathscr{O}_{1}^{\mathrm{R}}\right| K(0)\right\rangle_{m_{s}, m_{d}}=\left\langle 0\left|\mathscr{O}_{1}-\frac{c_{13}}{a^{2}} \mathscr{O}_{13}-\frac{c_{12}}{a} \mathscr{O}_{12}\right| K(0)\right\rangle_{m_{s}, m_{d}}=0 .
\end{aligned}
$$

In order to extract the coefficients $c_{13}$ and $c_{12}$ from these conditions we have computed for each ensemble the 2- and 3-pt meson correlators. Note that the non-perturbative determination of $c_{12}$, based on Eq. (4.2) is in progress. From the large time behavior of the 3-point correlators corresponding to the chromomagnetic and scalar density insertions one can compute $c_{13}$ in lattice units. In particular, from the following ratio:

$$
R_{3}\left(m_{s}, m_{l}\right) \equiv \frac{\left\langle\pi\left|\mathscr{O}_{1}\right| K\right\rangle}{\left\langle\pi\left|\mathscr{O}_{13}\right| K\right\rangle} \quad \text { and } \quad c_{13}=\lim _{m_{s}, m_{l} \rightarrow 0} a^{2} R_{3}\left(m_{s}, m_{l}\right)
$$

we can obtain an estimate of the mixing coefficient $c_{13}$ at each value of the lattice spacing. The results obtained for $a^{2} R_{3}\left(m_{l}, m_{l}\right)$ are collected in Fig. 1 and show an almost linear dependence on the light quark mass. Our final determinations of the mixing coefficient $c_{13}$ are reported in Table 3. Note that the total uncertainties on $c_{13}$ are of the order of $0.02 \%-0.1 \%$. The value which we obtained for $Z_{13}$ in one-loop perturbation theory leads to an estimate for $c_{13}$ :

$$
c_{13}^{\text {pert }}=a^{2} Z_{13}^{\text {pert }}=\frac{g^{2} C_{F}}{16 \pi^{2}}(36.0613)
$$

It can be seen that our non-perturbative determinations of $c_{13}$ differ by less than $\sim 10 \%$ from the 


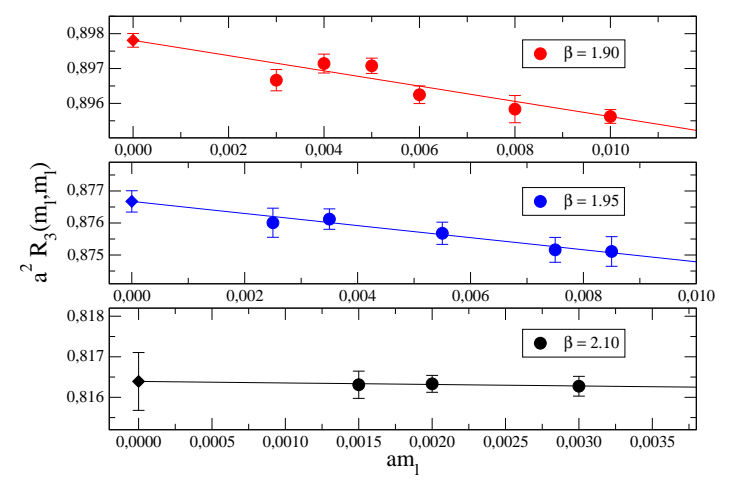

Figure 1: The ratio $a^{2} R_{3}\left(m_{l}, m_{l}\right)$ versus the (twisted) quark bare mass $a m_{l}$ for each value of the lattice spacing. The solid lines are the results of linear fits in $a m_{l}$ applied to the data. The diamonds represent the values of the mixing coeffcient $c_{13}$ obtained in the chiral limit (after Ref. [5]).

\begin{tabular}{|l|l|l|}
\hline$\beta$ & $c_{13}^{\text {pert }}$ & $c_{13}$ \\
\hline 1.90 & 0.96152 & $0.89769(17)$ \\
\hline 1.95 & 0.93686 & $0.87687(36)$ \\
\hline 2.10 & 0.86995 & $0.81646(78)$ \\
\hline
\end{tabular}

Table 3: Values of the mixing coefficient $c_{13}^{\text {pert }}$ obtained at one-loop in perturbation theory and $c_{13}$ obtained as the chiral limit at three values of the lattice spacing (see Fig.1).

perturbative predictions at one loop. For detailed information on non-perturbative results, see the proceedings by V. Lubicz in this conference [5].

Acknowledgments: The work of M. Constantinou and M. Costa was supported by the Cyprus

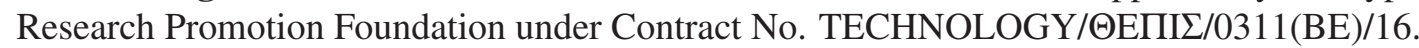

D. Meloni acknowledges MIUR (Italy) for financial support under the program Futuro in Ricerca 2010 (RBFR10O36O).

We also acknowledge the CPU time provided by the PRACE Research Infrastructure under the project PRA027 at JSC (Germany), and by the agreement between INFN and CINECA under the specific initiative INFN-lqcd123.

\section{References}

[1] R.Frezzotti and G. C. Rossi, JHEP 0410 (2004) 070, [arXiv:hep-lat/0407002].

[2] G. D’Ambrosio, G. Isidori, G. Martinelli, Phys. Let. B480 (2000) 164, [arXiv:hep-ph/9911522].

[3] L. Maiani, G. Martinelli and C. T. Sachrajda, Nucl. Phys. B368 (1992) 281.

[4] M. Constantinou, M. Costa, R. Frezzotti, V. Lubicz, G. Martinelli, D. Meloni, H. Panagopoulos and S. Simula, PoS LATTICE2013 (2013) 316, [arXiv:1311.5057].

[5] M. Constantinou, M. Costa, R. Frezzotti, V. Lubicz, G. Martinelli, D. Meloni, H. Panagopoulos and S. Simula, "Non-perturbative study of the chromagnetic operator on the lattice", PoS LATTICE2014. 